OPEN ACCESS

Edited by: Jon Pittman,

University of Manchester, UK

Reviewed by:

Ralph Henry,

University of Arkansas, USA

Bettina Bölter,

Ludwig-Maximilians-Universität

München, Germany

${ }^{*}$ Correspondence:

Danja Schünemann,

Molecular Biology of Plant Organelles,

Ruhr-University Bochum, Universitätsstraße 150, 44801

Bochum, Germany

danja.schuenemann@rub.de

Specialty section:

This article was submitted to Plant Traffic and Transport, a section of the

journal Frontiers in Plant Science

Received: 13 February 2015 Accepted: 27 March 2015

Published: 13 April 2015

Citation:

Walter B, Pieta T and Schünemann D (2015) Arabidopsis thaliana mutants lacking cpFtsY or cpSRP54 exhibit different defects in photosystem II repair. Front. Plant Sci. 6:250. doi: 10.3389/fpls.2015.00250

\section{Arabidopsis thaliana mutants lacking cpFtsY or cpSRP54 exhibit different defects in photosystem II repair}

\author{
Björn Walter ${ }^{1}$, Thomas Pieta ${ }^{2}$ and Danja Schünemann ${ }^{1 *}$ \\ ${ }^{1}$ Molecular Biology of Plant Organelles, Ruhr-University Bochum, Bochum, Germany, ${ }^{2}$ Plant Cell Physiology and Molecular \\ Biology, Ruhr-University Bochum, Bochum, Germany
}

Photosystem II (PS II) is a multi subunit protein complex embedded in the thylakoid membrane of cyanobacteria and chloroplasts. As the PS I| reaction center protein D1 is prone to a light induced damage that inhibits PS II function especially at elevated light intensities, a highly ordered repair process including synthesis, targeting and insertion of D1 has evolved. To elucidate the function of the chloroplast signal recognition particle subunits, cpSRP43 and cpSRP54, and the cpSRP-receptor cpFtsY in D1 biogenesis we investigated the efficiency of the PS II repair cycle in the corresponding mutants of Arabidopsis thaliana. Immunological analyses, PAM measurements and in vivo labeling experiments demonstrate an impaired replacement of damaged D1 in the cpftsy mutant, while the chaos and the ffc mutant lacking cpSRP43 and cpSRP54, respectively, were not or hardly affected. The defect in cpftsy was neither caused by an impaired $p s b A$ transcript accumulation, D1 translation initiation nor by an enhanced D1 degradation. Further experiments revealed a decreased amount of salt stable, thylakoid membraneassociated translating ribosomes in the cpftsy mutant, while the amount of membraneassociated translating ribosomes is unaltered in the chaos and the ffc mutants. Therefore, our data indicate that the lack of cpFtsY leads to an inefficient PS II repair cycle caused by an impaired binding of translating ribosomes to the thylakoid membrane.

Keywords: photosystem II, signal recognition particle, thylakoid membrane, protein transport, D1, photodamage

\section{Introduction}

The photosystem II (PS II) is a multi subunit protein pigment complex embedded in the thylakoid membrane of cyanobacteria and chloroplasts of algae and higher plants. There, it facilitates the light dependent charge separation and water splitting. The PS II subunits are encoded on the plastid and the nuclear genome. Nuclear encoded PS II subunits are synthesized in the cytosol and imported into the chloroplast posttranslationally (Schleiff and Becker, 2011; Paila et al., 2014). After the import into the stroma, the PS II subunits are transported across or inserted into the thylakoid membrane by four distinct mechanisms (Schünemann, 2007; Albiniak et al., 2012; Celedon and Cline, 2013). One of these is the posttranslational chloroplast signal recognition particle (cpSRP) dependent pathway that is responsible for the insertion of the light harvesting chlorophyll a/b binding proteins (LHCPs) that form the major antenna complexes of both photosystems. The cpSRP comprising the $43 \mathrm{kDa}$ subunit

Abbreviations: PS II, photosystem II; RNC, ribosome nascent chain complex; SRP, signal recognition particle. 
cpSRP43 and the universally conserved $54 \mathrm{kDa}$ subunit cpSRP54 binds the highly hydrophobic LHCPs to keep them soluble during the passage through the stroma. This transit complex is recruited to the thylakoid membrane by the membraneassociated receptor cpFtsY and the insertase Alb3, which is responsible for LHCP insertion (Richter et al., 2010). In contrast to nuclear encoded PS II subunits, plastid encoded polytopic PS II subunits are cotranslationally inserted into the thylakoid membrane. In Escherichia coli, translating ribosomes (RNCs) synthesizing polytopic inner membrane proteins are guided to the plasma membrane in a concerted process facilitated by the SRP consisting of the cpSRP54 homolog Ffh and the SRP RNA (Akopian et al., 2013). Ffh binds RNCs by a direct interaction with the ribosomes exit tunnel and the nascent polypeptide chain (Gu et al., 2003; Bornemann et al., 2008). Subsequently, this SRPRNC complex is guided to the SecYEG translocase by an interaction with the membrane associated receptor FtsY (Akopian et al., 2013; Denks et al., 2014). In chloroplasts, cpSRP54 interacts transiently with the nascent D1 chain (Nilsson et al., 1999; Nilsson and van Wijk, 2002) and D1 was shown to be inserted into the thylakoid membrane via the cpSec-translocon (Zhang et al., 2001). However, the chloroplast SRP system in higher plants differs significantly from the bacterial SRP-dependent transport as it does not contain a SRP-RNA, which is indispensable in all cytosolic SRP systems (Schünemann et al., 1998; Träger et al., 2012).

As the D1 protein is prone to a light induced damage, the replacement of D1 is a critical step during the PS II repair cycle to maintain its functionality (Kato and Sakamoto, 2009; Nixon et al., 2010). Previously, analyses of Arabidopsis thaliana mutants lacking cpSRP43 (chaos), cpSRP54 (ffc) and cpFtsY (cpftsy) demonstrated that chaos was characterized by a specific defect in accumulation of LHC proteins while ffc and cpftsy showed in addition a reduced steady state level of the reaction center proteins D1, PsaA and PsaB (Amin et al., 1999; Klimyuk et al., 1999; Tzvetkova-Chevolleau et al., 2007). However, cpftsy exhibited a significantly stronger phenotype than $f f c$ resulting in a strong chlorotic phenotype (TzvetkovaChevolleau et al., 2007) and seedling lethality (Asakura et al., 2004, 2008). Cpftsy showed also a more severe reduction of the maximal photochemical efficiency of PS II, which indicates a defect in proper assembly and activity of PS II, and a more rapid saturation of the photosynthetic electron transport by light (Tzvetkova-Chevolleau et al., 2007). Hence, biochemical data and mutant analysis indicate a role of cpSRP54 and cpFtsY in cotranslational insertion of D1 and other chloroplast encoded membrane proteins, but their individual relevance for this process and their precise molecular function is not clear yet.

In the present study, we compared the efficiency of D1 replacement during the PS II repair cycle in the cpSRP pathway mutants and wild type. Our data reveal a drastic defect of the cpftsy mutant in the PS II repair cycle, while the ffc mutant is only slightly affected. Additionally, the cpftsy mutant showed a reduced association of translating ribosomes with the thylakoid membrane indicating a disturbance in the binding of D1 translating ribosomes to the membrane.

\section{Material and Methods}

\section{Plant Materials and Growth Conditions}

The A. thaliana knock out mutants lacking cpSRP54 (ffc 1-2, referred to as $f f$ ) , cpSRP43 (chaos), and cpFtsY (cpftsy) were described previously (Amin et al., 1999; Klimyuk et al., 1999; Tzvetkova-Chevolleau et al., 2007). Mutant and wild type (ecotype Col-0) plants were grown on soil under artificial light $\left(8 \mathrm{~h}, 120 \mu \mathrm{mol}\right.$ photons $\left.\mathrm{m}^{-2} \mathrm{~s}^{-1}\right)$ and constant temperature of $22^{\circ} \mathrm{C} / 19.5^{\circ} \mathrm{C}$ (day/night) in GroBanks (CLF Plant Climatics).

\section{Protein Extraction, SDS-/BN-PAGE and Immunoblot Analysis}

Total protein extracts of rosette leaves were prepared using the peqGOLD TriFast reagent (Peqlab). The protein concentration of the extracts was determined using the BCA protein assay kit (Pierce). Proteins were separated on $12-15 \%$ SDS-polyacrylamide gels and subsequently coomassie stained or blotted onto nitrocellulose membrane (Macherey\&Nagel). Transferred proteins were detected by specific antibodies against D1 (Agrisera), Cytochrome f (Agrisera) and Rpl4 (Uniplastomic) and enhanced chemiluminescence reaction, ECL (Pierce).

BN-PAGE was conducted according to Schagger and von Jagow (1991) using the NativePAGE running buffer kit (Life Technologies). After BN-PAGE, the separated protein complexes were transferred to PVDF membrane (Macherey and Nagel) by western blot. Subsequently, the dye was removed from the membrane by incubation in methanol and the membrane was then used for immunodetection.

\section{Isolation of Thylakoid Membranes}

Leaves of $A$. thaliana in the rosette stage were homogenized in $50 \mathrm{mM}$ HEPES $\mathrm{pH} 8.0,330 \mathrm{mM}$ sorbitol, $15 \mathrm{mM}$ Mg-acetate (SHM) supplemented with $5 \mathrm{mM}$ ascorbate and $0.05 \%(\mathrm{w} / \mathrm{v})$ BSA using an Ultrathurrax (IKA). The homogenate was filtered through two layers of miracloth (Calbiochem). Afterwards, the chloroplasts were harvested by centrifugation $(1500 \mathrm{~g}, 5 \mathrm{~min}$, $4^{\circ} \mathrm{C}$ ) and washed in SHM. Then, chloroplasts were lysed in $20 \mathrm{mM}$ HEPES $\mathrm{pH}$ 8.0, $15 \mathrm{mM}$ Mg-acetate (HM) for $10 \mathrm{~min}$ on ice. After that, thylakoids were spun down $\left(1500 \mathrm{~g}, 5 \mathrm{~min}, 4^{\circ} \mathrm{C}\right.$ ) and the supernatant was discarded. For BN-PAGE analyses, the pellet was resuspended in $25 \mathrm{mM}$ BisTris- $\mathrm{HCl} \mathrm{pH} 7.0,30 \%(\mathrm{v} / \mathrm{v})$ glycerol supplemented with $1.5 \%(\mathrm{w} / \mathrm{v}) \beta$-D-dodecylmaltoside at a chlorophyll concentration of $1 \mathrm{mg} \mathrm{ml}^{-1}$ and incubated for $10 \mathrm{~min}$ on ice. After removal of insoluble material $(20,000 \mathrm{~g}$, $10 \mathrm{~min}, 4^{\circ} \mathrm{C}$ ), the supernatant was supplemented with coomassie G-250 (Life Technologies) to a final concentration of $0.25 \%$ (w/v) and used for BN-PAGE with subsequent western blot.

To analyze the membrane association of ribosomes, the isolated thylakoids were resuspended in HM at a chlorophyll concentration of $1 \mathrm{mg} \mathrm{ml}^{-1}$ and divided into five equal fractions. The thylakoids were spun down $\left(1500 \mathrm{~g}, 5 \mathrm{~min}, 4^{\circ} \mathrm{C}\right)$ and resuspended in $\mathrm{HM}$ supplemented with $60 \mathrm{mM} \mathrm{NaCl}, 1 \mathrm{M} \mathrm{NaCl}$, $50 \mathrm{mM}$ puromycin/60 mM NaCl, $50 \mathrm{mM}$ puromycin/1 $\mathrm{M} \mathrm{NaCl}$ or $0.2 \mathrm{M} \mathrm{Na}_{2} \mathrm{CO}_{3}$, respectively. After an incubation $\left(30 \mathrm{~min}, 37^{\circ} \mathrm{C}\right.$, rotating end over end), the thylakoids were sedimented and liberated from the supernatant. Finally, the pellets were resuspended 
in $\mathrm{HM}$ at a chlorophyll concentration of $2 \mathrm{mg} \mathrm{ml}^{-1}$ and 1:1 diluted by the subsequent addition of SDS sample buffer.

\section{Nucleic Acid Analysis}

The isolation of total RNA from $A$. thaliana leaf tissue was done according to US patent 5,973,137, Gentra Systems, Purescript ${ }^{\circledR}$ (Gentra-Systems Inc., Minneapolis, USA) and the concentration of RNA was determined with Nanodrop 2000c (Peqlab). For northern blot analyses, $3 \mu \mathrm{g}$ total RNA were loaded on each lane and separated on a denaturating 1.2\% (w/v) agarose gel. Subsequently, RNA was transferred onto a positively charged nylon membrane (Roth) and $p s b A$ transcript was detected with a specific probe.

For production of the $p s b A$ probe, the template vector pGEMT Easy psbA (Loschelder et al., 2006) was linearized with PstI and used for in vitro transcription (Promega T7 transcription kit) and labeled with digoxygenin (DIG) according to the Kit manual (Roche). Hybridized probes were detected using a DIG-specific, alkaline phosphatase conjugated antibody (Roche). Chemiluminescence was detected by exposure to a film (Fuji).

Polysomes were isolated from A. thaliana leaves according to Barkan (1993). Leaf tissue (400 mg) was frozen in liquid nitrogen and homogenized with a mortar and a pestle. Membranes were subsequently solubilized with $1 \%(\mathrm{v} / \mathrm{v})$ Triton X-100 and $2 \%(\mathrm{v} / \mathrm{v})$ polyoxyethylene (10) tridecyl ether. Microsomal membranes were then solubilized with $0.5 \%(\mathrm{w} / \mathrm{v})$ sodiumdeoxycholate and insoluble material was removed $(15,000 \mathrm{~g}, 15 \mathrm{~min}$, $4{ }^{\circ} \mathrm{C}$ ). The supernatant was then loaded onto a $15-55 \%$ sucrose step gradient and centrifuged $\left[250,000 \mathrm{~g}, 65 \mathrm{~min}, 4^{\circ} \mathrm{C}\right.$, TST 60.4 rotor (Kontron)]. The gradient was fractionated into 14 equal fractions and each fraction was subjected to RNA extraction with phenol-chloroform two times. After ethanol precipitation, the RNA was subjected to northern blot analysis as described above. To mimic the situation of impaired translation initiation of $p s b A$ mRNA, the polysomes were disrupted according to $\mathrm{Lu}$ and Hanson (1996).

\section{Chlorophyll Fluorescence Analysis}

The maximum quantum efficiency $\mathrm{F}_{\mathrm{v}} \mathrm{F}_{\mathrm{m}}^{-1}$ of intact leaves of A. thaliana null mutants and Col-0 was determined using a portable PAM-2000 (Heinz Walz GmbH, Germany, www.walz. com). Before measurements, each plant was dark adapted for $15 \mathrm{~min}$.

\section{In vivo Labeling of Proteins and Pulse-chase Experiments}

The de novo synthesis of thylakoid membrane proteins was monitored as described previously (Lennartz et al., 2001) with the following modifications. Leaf discs of $A$. thaliana plants in rosette stage where prepared using a cork drill $(0.5 \mathrm{~cm}$ diameter $)$. The leaf discs were incubated for $30 \mathrm{~min}$ at $\mathrm{RT}$ in preparation buffer (50 mM Tris- $\mathrm{HCl} \mathrm{pH} \mathrm{7.5,} 0.2 \mathrm{mg} \mathrm{ml}^{-1}$ cycloheximide and $0.1 \%$ (v/v) Tween-20). Subsequently, the leaf discs were transferred to labeling buffer $\left(50 \mathrm{mM}\right.$ Tris- $\mathrm{HCl} \mathrm{pH} 7.5,0.1 \mathrm{mg} \mathrm{ml}^{-1}$ cycloheximide, $0.1 \%(\mathrm{v} / \mathrm{v})$ Tween-20 and $0.1 \mu \mathrm{Ci} \mu^{-1}\left[{ }^{35} \mathrm{~S}\right]$-methionine (specific activity $>1000 \mathrm{Ci} \mathrm{mmol}^{-1}$, Hartmann Analytic) and incubated at $25^{\circ} \mathrm{C}$ for $60 \mathrm{~min}$ at indicated light intensities. After washing the leaf discs five times with washing buffer $(50 \mathrm{mM}$ Tris- $\mathrm{HCl} \mathrm{pH} 7.5,750 \mathrm{mM} \mathrm{NaCl}, 2 \mathrm{mM}$ EDTA), the tissue was homogenized with a steel pestle in $300 \mu \mathrm{l}$ homogenization buffer (50 mM Tris- $\mathrm{HCl}$ pH 7.5, $150 \mathrm{mM} \mathrm{NaCl}, 2 \mathrm{mM}$ EDTA). Next, membranes were pelleted $\left(15,000 \mathrm{~g}, 10 \mathrm{~min}, 4^{\circ} \mathrm{C}\right)$ and washed two times. Subsequently, membranes were resuspended in $50 \mu \mathrm{l}$ homogenization buffer supplemented with $2 \%(\mathrm{w} / \mathrm{v})$ SDS and incubated for $10 \mathrm{~min}$ at $25^{\circ} \mathrm{C}$ with a following heating at $70^{\circ} \mathrm{C}$ for $2 \mathrm{~min}$. After centrifugation ( $10 \mathrm{~min}, 15,000 \mathrm{~g}$, RT), the supernatant was transferred to a new reaction tube. The supernatant was used for chlorophyll determination and SDS-PAGE and radiolabeled proteins were detected by autoradiography.

For pulse-chase experiments, chloroplast proteins in leaf discs were pulse labeled as described above for $60 \mathrm{~min}$ followed by a chase time in $10 \mathrm{mM}$ non-labeled L-methionine for $8 \mathrm{~h}$ at $150 \mu \mathrm{mol}$ photons $\mathrm{m}^{-2} \mathrm{~s}^{-1}$. Every hour, five leaf discs were frozen in liquid nitrogen and homogenized in homogenization buffer supplemented with $1 \mathrm{M} \mathrm{NaCl}$. After centrifugation, the membrane proteins were SDS-solubilized in $40 \mu \mathrm{l}$ homogenization buffer as described above. Subsequently, $15 \mu \mathrm{l}$ were used for SDS-PAGE.

\section{Quantification}

Signals of western and northern blots and radiolabeled proteins were quantified using ImageJ (http://imagej.nih.gov/ij).

\section{Results}

\section{The cpftsy Mutant is Drastically Impaired in PS II Repair while the ffc Mutant is only Slightly Affected}

To examine the role of cpSRP43, cpSRP54, and cpFtsY in the biogenesis of plastid-encoded D1, we initially compared the D1 protein level in the chaos, $f f c$, and cpftsy mutants and wild type plants after growth at normal light conditions $(120 \mu \mathrm{mol}$ photons $\left.\mathrm{m}^{-2} \mathrm{~s}^{-1}\right)$, high light stress $\left(1000 \mu \mathrm{mol}\right.$ photons $\left.\mathrm{m}^{-2} \mathrm{~s}^{-1}\right)$ and a following recovery phase at low light conditions $(50 \mu \mathrm{mol}$ photons $\mathrm{m}^{-2} \mathrm{~s}^{-1}$ ) by western blot analysis (Figure 1). The D1 protein level decreased drastically after growth at high light conditions to about $30-40 \%$ of the initial protein level in all analyzed plant lines. After the recovery phase the amount of D1 in the wild type and the chaos mutant recovered completely. Interestingly, the D1 protein level in the $f f c$ mutant recovered up to $90 \%$ of the initial level, while the D1 protein level in cpftsy showed no recovery.

As the de novo insertion of D1 is a major step in the maintenance of PS II, we used pulse amplitude modulation (PAM) measurements to analyze chlorophyll a fluorescence of P680 in order to examine the maximum quantum efficiency $\left(\mathrm{F}_{\mathrm{v}} \mathrm{F}_{\mathrm{m}}^{-1}\right)$, an indicator for PS II functionality. To this end, wild type and mutant plants were sequentially exposed to normal light, high light and low light. Prior to each measurement, plants were dark adapted for $15 \mathrm{~min}$. The PAM measurements revealed a decline of maximum quantum efficiency after exposure to high light in all analyzed plants. After the subsequent low light period, the maximum quantum efficiency in wild type and chaos recovered almost completely to about $90 \%$ of the initial value. In agreement with our previous observation, the recovery efficiency in $f f c$ and 


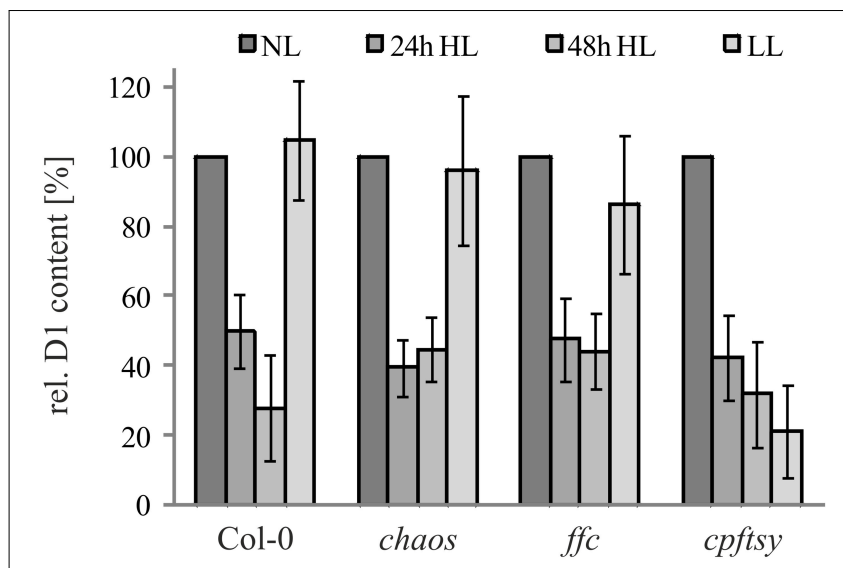

FIGURE 1 | D1 level in cpSRP mutant plants under high light conditions and a subsequent recovery phase. Wild type and cpSRP mutant plants were raised at normal light conditions ( $\mathrm{NL}, 120 \mu \mathrm{mol}$ photons $\mathrm{m}^{-2} \mathrm{~s}^{-1}$ ) to rosette stage and then exposed to high light $\left(\mathrm{HL}, 1000 \mu \mathrm{mol}\right.$ photons $\mathrm{m}^{-2}$ $\mathrm{s}^{-1}$ ) for 24 or $48 \mathrm{~h}$. Subsequently, plants were exposed to low light for $48 \mathrm{~h}$ (LL, $50 \mu \mathrm{mol}$ photons $\cdot \mathrm{s}^{-1} \cdot \mathrm{m}^{-2}$ ). Total protein extracts from $100 \mathrm{mg}$ fresh weight of intact and fully expanded leaves were prepared after each light treatment and used for immunodetection of D1. The signals of the D1 immunoblots were quantified using the ImageJ program. The D1 levels in normal light conditions were set to $100 \%$. Error bars indicate the standard deviation $(n=4)$.

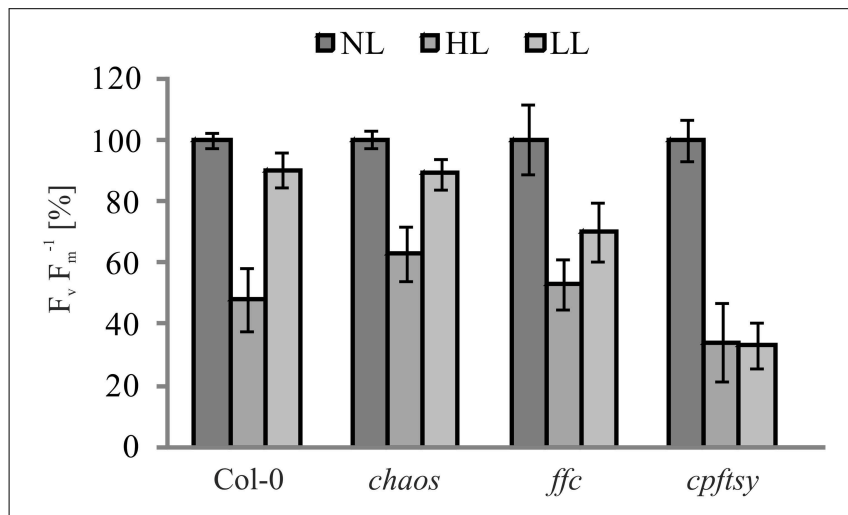

FIGURE 2 | Maximum quantum efficiency of PS II in cpSRP mutants. The maximum quantum efficiency of $P S \|\left(F_{v} F_{m}^{-1}\right)$ was determined in wild type and mutant plants in the rosette stage after dark adaption. Prior to the measurements, plants were raised under normal light conditions (NL, $120 \mu \mathrm{mol}$ photons $\mathrm{m}^{-2} \mathrm{~s}^{-1}$ ), then exposed to high light for $180 \mathrm{~min}\left(\mathrm{HL}, 1000 \mu \mathrm{mol}\right.$ photons $\mathrm{m}^{-2} \mathrm{~s}^{-1}$ ) with a following regeneration at low light for $90 \mathrm{~min}$ (LL, $50 \mu \mathrm{mol}$ photons $\mathrm{m}^{-2} \mathrm{~s}^{-1}$ ). For each plant, $\mathrm{F}_{\mathrm{v}} \mathrm{F}_{\mathrm{m}}^{-1}$ values at $\mathrm{NL}$ were adjusted to $100 \%$. The error bars show the standard deviation $(n=10)$.

cpftsy differed significantly. While the $f f c$ mutant showed a recovery of up to $70 \%$ of its initial value, the cpftsy mutant showed no recovery of the maximum quantum efficiency (Figure 2).

Growth at high light conditions is accompanied by a higher synthesis rate of D1 to enable an effective PS II repair cycle. To compare the ability of the cpSRP mutants and the wild type to increase the D1 synthesis rate at high light intensities, leaf discs were incubated in a medium containing radiolabeled methionine at low and higher light intensities of 50 and $400 \mu \mathrm{mol}$ photons

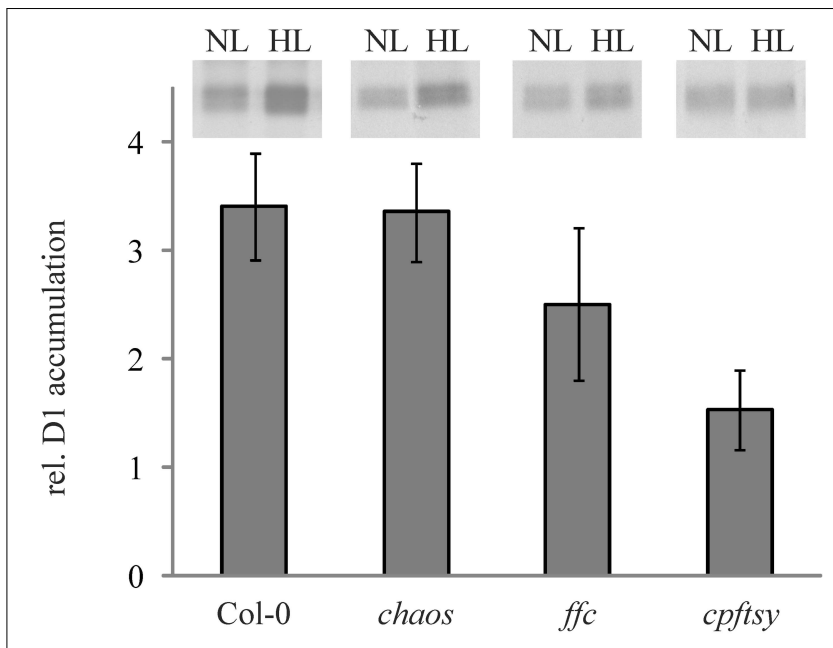

FIGURE 3 | In vivo synthesis ratio of [ ${ }^{35}$ S]-labeled D1 in wild type and cpSRP mutant plants at high light compared to low light. Leaf discs of wild type and cpSRP mutant plants were incubated in [ $\left.{ }^{35} \mathrm{~S}\right]$ methionine containing solution at low light $\left(50 \mu \mathrm{mol}\right.$ photons $\left.\mathrm{m}^{-2} \mathrm{~s}^{-1}\right)$ and higher light $\left(400 \mu \mathrm{mol}\right.$ photons $\left.\mathrm{m}^{-2} \mathrm{~s}^{-1}\right)$ after preincubation in cycloheximide for $60 \mathrm{~min}$. Subsequently, thylakoid membrane proteins were extracted and used for SDS-PAGE and autoradiography (equal amounts of chlorophyll were loaded). At the top, representative autoradiographs of radiolabeled D1 are depicted. The signals were subsequently quantified using the ImageJ program and the higher light values were related to low light values. A value of 1 indicates that no elevated D1 accumulation was observed under higher light conditions in comparison to low light. The error bars show the standard deviation $(n=4)$.

$\mathrm{m}^{-2} \mathrm{~s}^{-1}$, respectively. As shown in Figure 3, wild type and chaos accumulated about 3.5 times more radiolabeled D1 at elevated light intensities in comparison to low light, while the D1 synthesis in $f f c$ was elevated 2.5 fold compared to low light. In contrast, cpftsy showed only a very slight increase by a factor of 1.5 in D1 accumulation.

To further analyze the role of the cpSRP components in D1 biogenesis, we investigated the ability of the chaos, ffc, and cpftsy mutants and the wild type to maintain high molecular weight PS II-LHC II supercomplexes at elevated light conditions. To this end, we conducted BN-PAGE analyses of thylakoid membrane protein complexes of plants exposed to normal light conditions, high light intensities and a subsequent recovery at low light conditions. The separated thylakoid membrane protein complexes were subsequently analyzed immunologically to detect D1 protein containing complexes. After growth at normal light conditions, clear signals of PS II-LHC II supercomplexes were detected in chaos, $f f c, c p f t s y$, and wild type plants (Figure 4). However, the composition of the supercomplexes differed in chaos and cpftsy from the wild type, which is likely due to variable compositions of the antenna complexes caused by the impact of the mutations on posttranslational LHCP transport. In addition, we observed a difference between the supercomplexes of cpftsy compared to chaos indicating an additional defect in PS II-LHC II biogenesis in cpftsy. Notably, the most drastic difference in PS II-LHC II supercomplex composition was observed at high light conditions between cpftsy and the other plant lines. At high light, the PS IILHC II supercomplexes in wild type, chaos and ffc were hardly 


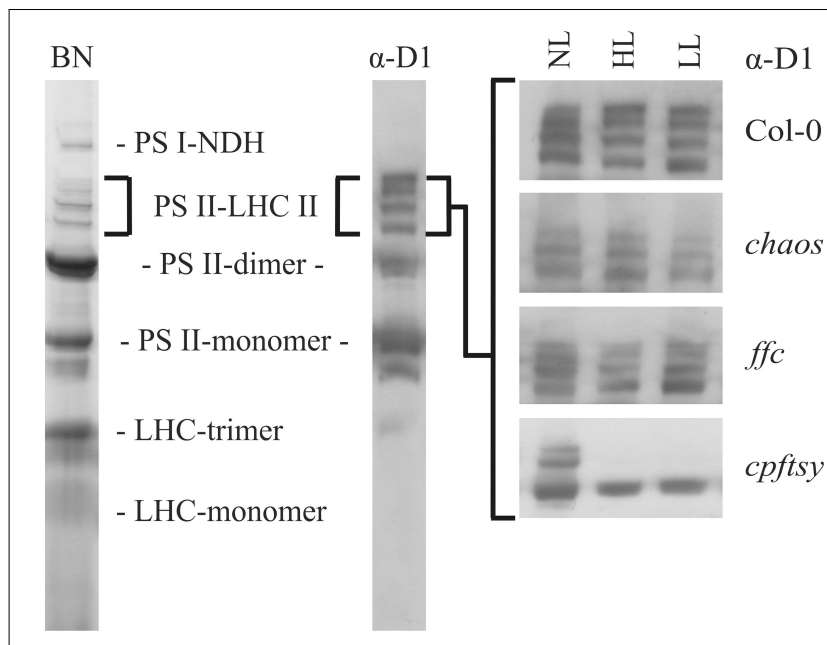

FIGURE 4 | Abundance of PS II-LHC II supercomplexes in wild type and cpSRP mutants at various light conditions. Thylakoid membrane protein complexes of wild type and cpSRP mutants were solubilized with $1.5(\mathrm{w} / \mathrm{v})$ $\beta$-D-dodecylmaltoside after growth at normal light (NL, $120 \mu \mathrm{mol}$ photons $\left.\mathrm{m}^{-2} \mathrm{~s}^{-1}\right)$, high light ( $\mathrm{HL}, 1000 \mu \mathrm{mol}$ photons $\mathrm{m}^{-2} \mathrm{~s}^{-1}$ for $16 \mathrm{~h}$ ) and a subsequent regeneration at low light (LL, $50 \mu \mathrm{mol}$ photons $\mathrm{m}^{-2} \mathrm{~s}^{-1}$ for $8 \mathrm{~h}$ ). Of each sample, an equal amount of chlorophyll $(1.5 \mu \mathrm{g})$ was separated by BN-PAGE and blotted onto PVDF membrane. The amount of PS II-LHC II super complexes was analyzed using a D1 specific antibody. affected. Contrary, the cpftsy mutant showed a drastic reduction of PS II-LHC II supercomplexes without any regeneration during subsequent exposure to low light.

Taken together, our data provide several lines of evidence indicating a severely reduced PS II repair efficiency in $c p f t s y$, while $f f c$, and chaos are only slightly and not affected, respectively.

\section{psbA Transcript Accumulation, Polysome Formation and D1 Stability are not Affected in the cpftsy Mutant}

The reduced repair of damaged D1 in cpftsy suggested a possible defect in the D1 targeting or insertion mechanism. However, to rule out a high light dependent decline of the $p s b A$ transcript abundance, the $p s b A$ transcript level was analyzed in chaos, $f c$, cpftsy, and wild type plants treated in the same way as for the investigation of the D1 protein level described in Figure 1. As shown in Figure 5, the $p s b A$ transcript levels of all analyzed plants were comparable under normal light and no significant changes in high light and a subsequent recovery phase were observed. Therefore, these data clearly demonstrate that the reduced PS II repair cycle in cpftsy is not due to a change in $p s b A$ transcript level.

Next, we analyzed the formation of $p s b A$ mRNA-associated plastid polysomes to exclude an impaired translation initiation of $p s b A$ mRNA. To this end, a total leaf extract of each

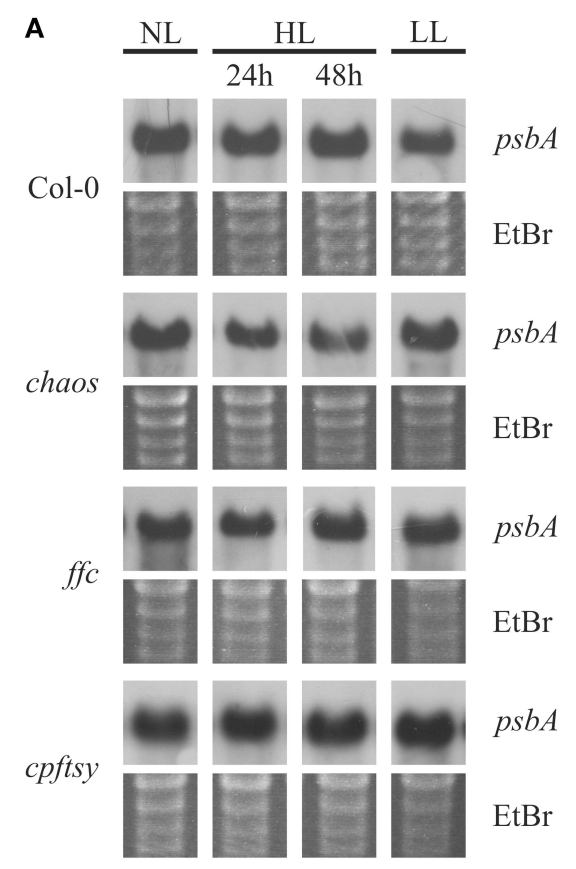

FIGURE 5 | PsbA transcript levels during high light stress treatment and a recovery phase. (A) Total RNA was isolated from fully expanded leaves of wild type and cpSRP mutant plants after growth at normal light $\left(120 \mu \mathrm{mol}\right.$ photons $\left.\mathrm{m}^{-2} \mathrm{~s}^{-1}\right), 24 \mathrm{~h}$ of high light $\left(1000 \mu \mathrm{mol}\right.$ photons $\left.\mathrm{m}^{-2} \mathrm{~s}^{-1}\right), 48 \mathrm{~h}$ of high light and a subsequent regeneration for $48 \mathrm{~h}$ at low light $\left(50 \mu \mathrm{mol}\right.$ photons $\mathrm{m}^{-2}$

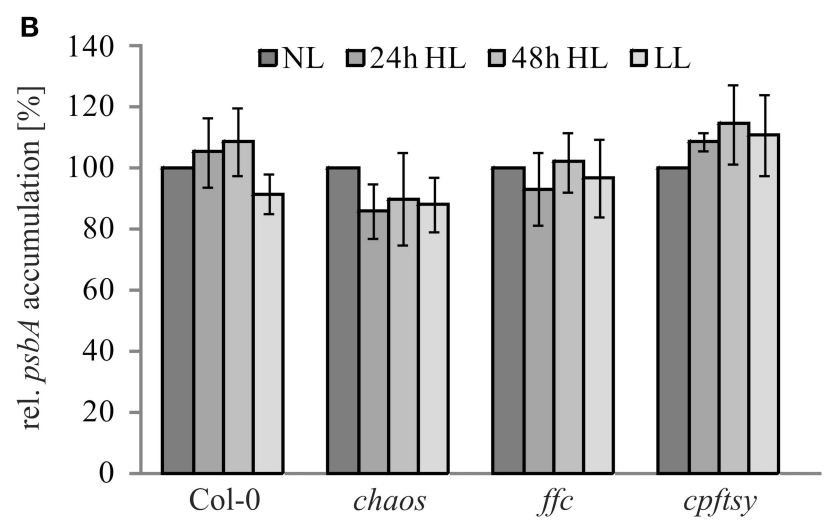

$\left.\mathrm{s}^{-1}\right)$. $3 \mu \mathrm{g}$ of the total RNA was used for northern blot analysis to investigate the amount of $p s b A$ transcript. As a loading control, gels were stained with ethidiumbromide. (B) Northern blot signals of $p s b A$ transcript were quantified using the ImageJ program. The $p s b A$ levels at normal light conditions were adjusted to $100 \%$. The error bars indicate the standard deviation $(n=3)$. 
plant line was loaded onto sucrose density gradients. Northern blot analysis of the fractionated gradient loaded with wild type extract showed that $p s b A$ mRNA entered fractions of higher sucrose concentration indicating an association with polysomes (Figure 6). To mimic the situation of an impaired translation reaction, a wild type leaf extract was prepared in presence of EDTA that leads to disassembly of ribosomes by depletion of $\mathrm{Mg}^{2+}$ (Yamamoto et al., 2010). In the corresponding sucrose density gradient, $p s b A$ mRNA was only detectable in fractions of low density. This reflects the situation in an A. thaliana mutant lacking the HCF173 protein that was shown to be essential for $p s b A$ translation initiation (Schult et al., 2007). The analyses of leaf extracts from the chaos, ffc and cpftsy mutants revealed a wild type-like distribution of $p s b A$ mRNA in the gradients for chaos and $c p f t s y$. The $f f c$ mutant exhibits differences in the distribution of $p s b A$ mRNA in fractions of higher sucrose density as less transcript was detected in these fractions (Figure 6). As it was shown previously that cpSRP54 is a component of D1-translating ribosomes, the lack of cpSRP54 might lead to a marked reduction in the molecular weight of heavy polysomes. In conclusion our data demonstrate a functional formation of polysomes and therefore an undisturbed translation initiation of $p s b A$ in chaos and cpftsy and indicate that this is also the case in $f f c$.

Next, we aimed to analyze whether the cpftsy mutant exhibits a possible enhanced degradation of D1. Therefore, pulse-chase experiments were conducted to analyze the half-time of the D1 protein in chaos, ffc, and cpftsy mutant. For this purpose, leaf discs of the mutant and wild type plants were incubated at low light conditions in a solution containing radiolabeled methionine and cycloheximide to label newly synthesized D1 protein and block the cytosolic translation machinery, respectively. As shown in Figure 7, clear signals of the D1 protein were still detected after a chase time of $8 \mathrm{~h}$ in the wild type indicating a rather high stability of the D1 protein. A similar high stability of the D1 protein was observed in the chaos and cpftsy mutants. The degradation pattern in the $f f c$ mutant indicates a slightly reduced stability after $6 \mathrm{~h}$ of chase incubation. As our data demonstrate a high stability of the D1 protein in the cpftsy mutant, an enhanced D1 degradation as an explanation for the poor PS II repair cycle in cpftsy can be excluded.

\section{The cpftsy Mutant is Impaired in Binding of Translating Ribosomes to the Thylakoid Membrane}

Since bacterial FtsY is essential for the targeting of translating ribosomes to the plasma membrane (Seluanov and Bibi, 1997; Herskovits and Bibi, 2000), it was conceivable that cpFtsY might play an important role in guiding translating ribosomes to the translocon in the thylakoid membrane. Initially, we compared the amount of total ribosomes in thylakoid membranes of the cpSRP-mutants and wild type, that were purified under lowsalt conditions $(60 \mathrm{mM} \mathrm{NaCl})$ by western blot analysis using an anti-Rpl4 antibody. As shown in Figure 8A, the analyzed plants exhibit a comparable amount of plastid ribosomes in the thylakoid membrane fraction. The equal loading was confirmed using an antibody raised against Cytochrome $\mathrm{f}$ that was shown to be imported into the thylakoid lumen independent

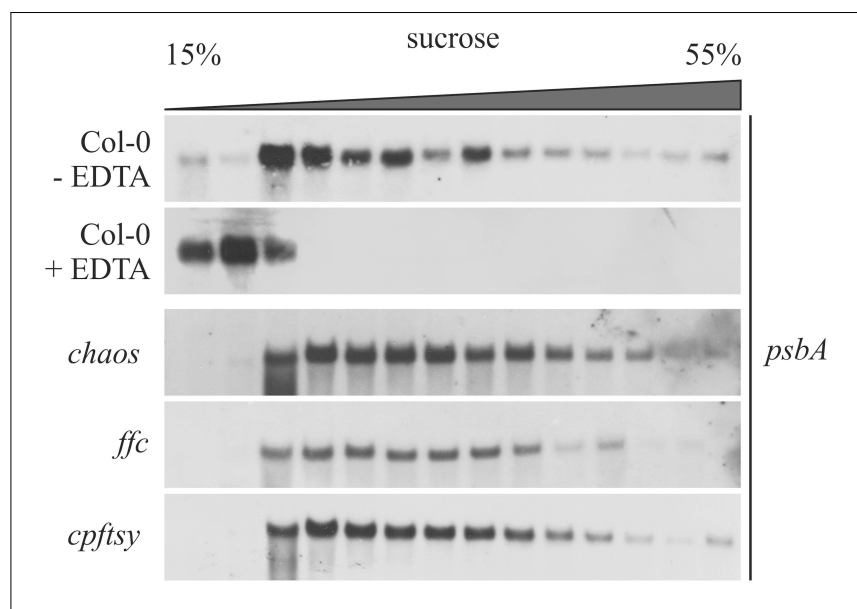

FIGURE 6 | Association of psbA transcript with chloroplast polysomes. Polysomes isolated from wild type (Col-0) and mutant plants at rosette stage were fractionated on a $15-55 \%$ sucrose gradient by ultracentrifugation. Subsequently, the gradients were fractionated and the amount of $p s b A$ transcript was investigated in each fraction by northern blot analyses using a psbA-specific probe. As a control, polysomes were destroyed using EDTA.

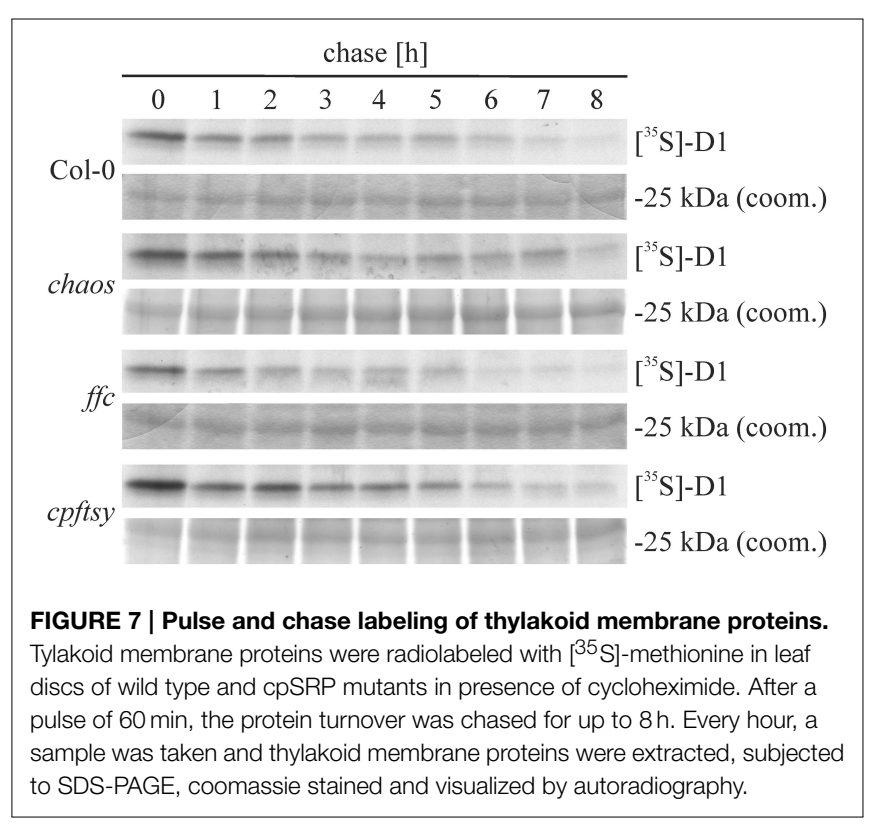

of cpSRP (Röhl and van Wijk, 2001). To compare the amount of membrane-bound translating ribosomes in wild type and the cpSRP mutants isolated thylakoid membranes were washed with buffers containing $60 \mathrm{mM}$ or $1 \mathrm{M} \mathrm{NaCl}$ with or without $50 \mathrm{mM}$ puromycin. As shown in Figure 8B, the ribosomes in wild type, chaos and ffc could only be removed from the membrane by washing the membranes with a combination of high salt and puromycin. Washing with either $1 \mathrm{M} \mathrm{NaCl}$ or $50 \mathrm{mM}$ puromycin did not reduce the amount of bound ribosomes. This indicates that these ribosomes are attached to the membrane by electrostatic interactions and the cotranslational insertion of nascent polypeptides into the membrane. In contrast, about 40 


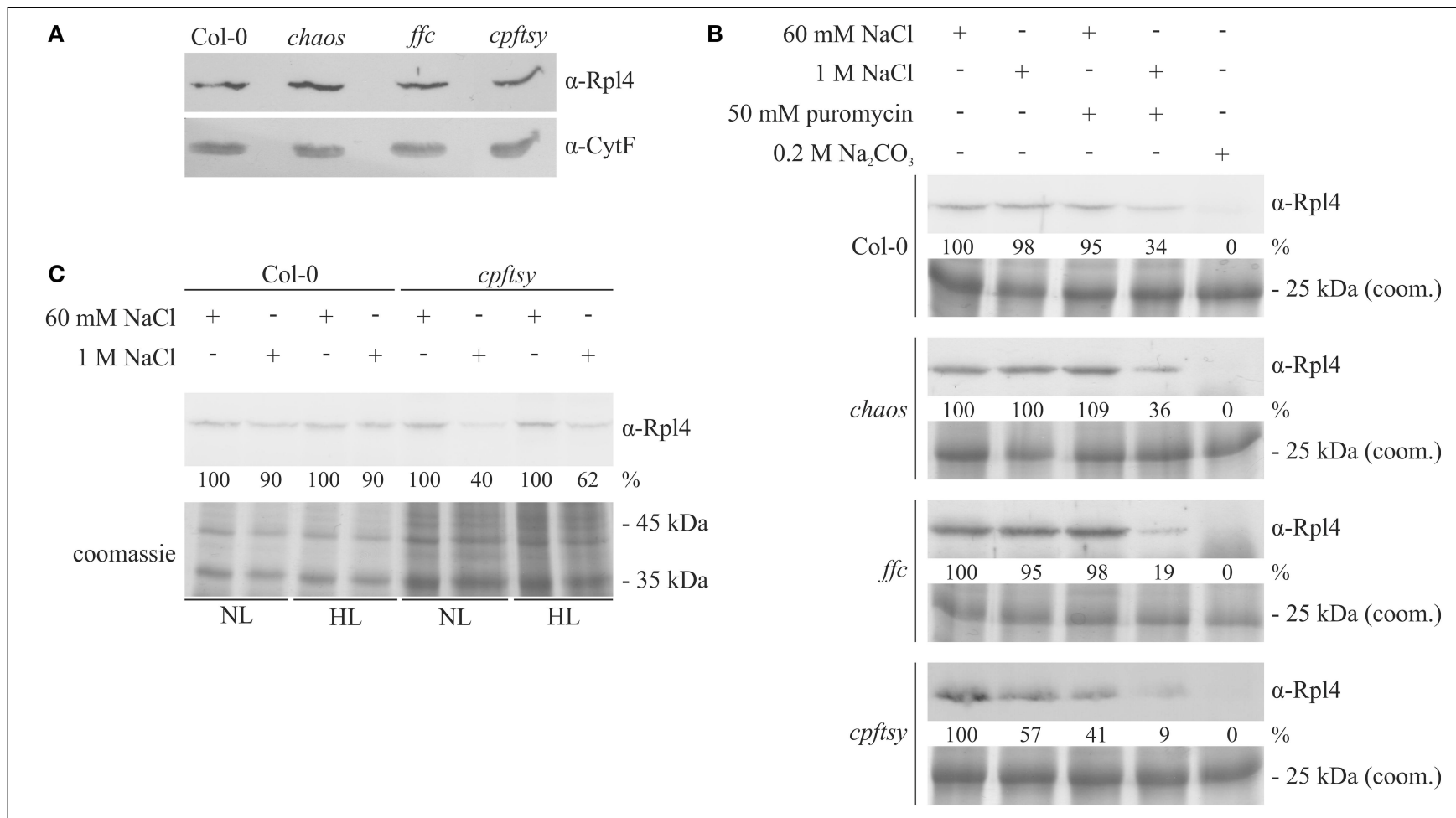

FIGURE 8 | Detection of salt-resistant and thylakoid

membrane-associated ribosomes. (A) Thylakoid membranes were isolated from wild type and the cpSRP-mutant plants grown at normal light intensity $\left(120 \mu \mathrm{mol}\right.$ photons $\left.\mathrm{m}^{-2} \mathrm{~s}^{-1}\right)$. Subsequently, the thylakoids were treated with $60 \mathrm{mM} \mathrm{NaCl}$. Afterwards, a sample corresponding to $15 \mu \mathrm{g}$ chlorophyll was subjected to SDS-PAGE with subsequent western blot. The amount of membrane-associated ribosomes was determined using an antibody raised against Rpl4. To control the equal loading, the amount of the luminal protein Cytochrome $f$ was investigated. (B) Thylakoid membranes were isolated from normal light $(120 \mu \mathrm{mol}$ photons $\mathrm{m}^{-2} \mathrm{~s}^{-1}$ ) adapted wild type and cpSRP mutants. Subsequently, the thylakoids were divided in 5 equal fractions and treated with washing conditions as indicated above. Afterwards, a sample corresponding to $15 \mu \mathrm{g}$ chlorophyll after each washing condition was separated by $12 \%$ SDS-PAGE. Finally, the proteins were transferred onto nitrocellulose membranes and the large ribosomal subunit protein Rpl4 was detected using a specific antibody. The signals were quantified using the ImageJ program. To control the equal loading, thylakoid membrane proteins corresponding to $7.5 \mu \mathrm{g}$ chlorophyll were separated by SDS-PAGE and visualized by coomassie staining. (C) Wild type and cpftsy plants were exposed for $8 \mathrm{~h}$ to normal light $\left(120 \mu \mathrm{mol}\right.$ photons $\left.\mathrm{m}^{-2} \mathrm{~s}^{-1}\right)$ and high light conditions $\left(1000 \mu \mathrm{mol}\right.$ photons $\left.\mathrm{m}^{-2} \mathrm{~s}^{-1}\right)$. Subsequently, thylakoid membranes were isolated and washed with lysis buffer supplemented with $60 \mathrm{mM} \mathrm{NaCl}$ or $1 \mathrm{M} \mathrm{NaCl}$. Thylakoid membrane proteins corresponding to $15 \mu \mathrm{g}$ chlorophyll were separated by $12 \%$ SDS-PAGE, transferred onto nitrocellulose and the ribosomal Rpl4 protein was detected using a specific antibody. The signals were quantified using the ImageJ program. To control the equal loading, thylakoid membrane proteins corresponding to $7.5 \mu \mathrm{g}$ chlorophyll were separated by SDS-PAGE and visualized by coomassie staining. or $60 \%$ of the ribosomes in cpftsy could be detached from the membrane by washing with high salt or puromycin, respectively. This indicates that the ribosomes in cpftsy, which were copurifed with thylakoids under low-salt conditions, are largely inactive or non-membrane protein translating ribosomes and are therefore less tightly bound. In all analyzed plants, ribosomes as peripheral components were efficiently removed by $0.2 \mathrm{M}$ $\mathrm{Na}_{2} \mathrm{CO}_{3}$.

Next, we aimed to dissect the abundance of membrane protein translating ribosomes at the thylakoid membrane after exposure to high light conditions. Therefore, thylakoid membranes were isolated from cpftsy and wild type plants exposed to normal and high light and washed with $60 \mathrm{mM} \mathrm{NaCl}$ or $1 \mathrm{M} \mathrm{NaCl}$. At both light conditions immunological analyses revealed a decrease of membrane-bound ribosomes after high salt treatment to $40-60 \%$ of the initial level exclusively in the cpftsy mutant, while the wild type retained $90 \%$ of the initial level (Figure 8C).

\section{Discussion}

In this study, the question regarding the role of cpSRP43, cpSRP54 and cpFtsY during the PS II repair cycle was addressed using corresponding mutants of $A$. thaliana. Therefore, the mutant plants were exposed to various light conditions and the efficiency of PS II maintenance was monitored. A drastic limitation of the PS II repair cycle in the cpftsy mutant was observed that was neither due to an impaired $p s b A$ mRNA transcript accumulation and translation initiation nor to an enhanced D1 degradation. Instead, the amount of salt-resistant membraneassociated ribosomes was decreased in the cpftsy mutant. The chaos mutant and, notably, the $f f c$ mutant showed no or only minor defects in PS II maintenance, respectively.

Based on the previous findings (1) that the $f f c$ mutant shows a reduced level of the chloroplast encoded photosystem I and II reaction center proteins (Amin et al., 1999), (2) that cpSRP54 
interacts transiently with the nascent chain of D1 when it emerges from the ribosome exit tunnel (Nilsson et al., 1999; Nilsson and van Wijk, 2002), (3) that one pool of cpSRP54 is associated with chloroplast ribosomes (Franklin and Hoffman, 1993; Schünemann et al., 1998) and (4) that cpSRP54 shows a strong homology to bacterial Ffh (Franklin and Hoffman, 1993), it has been assumed that cPSRP54 has a role in cotranslational protein insertion into the thylakoid membrane.

However, the lack of cpSRP54 in A. thaliana leads to a mild phenotype which is most visible at the young seedling stage when the first true leaves are yellowish and the subset of photosystem proteins that is reduced in the young leaves recovers to wild type level in the older leaves (Amin et al., 1999). Therefore, these data argue against a general important role of cpSRP54 in cotranslational protein insertion, although it cannot be ruled out that the loss of cpSRP54 in the ffc mutant is compensated by e.g., the upregulation of stromal chaperones as shown for ClpC (Rutschow et al., 2008). The present report extended the analysis of the role of cPSRP54 in cotranslational protein insertion by focusing on the PS II repair cycle during high light conditions. As it seems rather unlikely that the lack of cpSRP can be almost fully compensated by an upregulation of an alternative mechanisms under these stress conditions, our data indicate that cPSRP54 has no critical role in PS II maintenance. However, as there are several lines of evidence indicating a role of cpSRP54 in D1 biogenesis it might be possible that cpSRP54 has its major role in the de novo synthesis of photosynthetic protein complexes during leaf development.

Our data reveal a drastic disturbance of the PS II repair cycle due to the lack of the SRP-receptor cpFtsY. While the common model of bacterial SRP-mediated protein insertion proposes that ribosomes and transcripts are cotargeted to the membrane, where FtsY mediates the docking of the SRP-RNC complex to the SecYEG translocase (Luirink et al., 2005; Akopian et al., 2013), an alternative model is discussed in which ribosomes and transcripts are targeted separately to the membrane (Bibi, 2012). Here, FtsY serves as an anchor for ribosomes at the plasma membrane. Inner membrane protein coding mRNAs are delivered to the ribosome in a translation independent manner. Afterwards, the translation process is initiated. In a following step, SRP binds to the nascent chain and facilitates the insertion of the membrane protein via the Sec-translocase (Bibi, 2012). This model is in line with the finding that FtsY mutants exhibit a reduced number of

\section{References}

Akopian, D., Shen, K., Zhang, X., and Shan, S. O. (2013). Signal recognition particle: an essential protein-targeting machine. Annu. Rev. Biochem. 82, 693-721. doi: 10.1146/annurev-biochem-072711-164732

Albiniak, A. M., Baglieri, J., and Robinson, C. (2012). Targeting of lumenal proteins across the thylakoid membrane. J. Exp. Bot. 63, 1689-1698. doi: 10.1093/jxb/err444

Amin, P., Sy, D. A., Pilgrim, M. L., Parry, D. H., Nussaume, L., and Hoffman, N. E. (1999). Arabidopsis mutants lacking the 43- and 54-kilodalton subunits of the chloroplast signal recognition particle have distinct phenotypes. Plant Physiol. 121, 61-70. doi: 10.1104/pp.121.1.61 membrane-associated ribosomes, while this was not observed in Ffh-depleted cells (Herskovits and Bibi, 2000). Interestingly, our data also indicate a crucial role of cpFtsY for the association of plastid polysomes with the thylakoid membrane while cpSRP54 seems to be dispensable for this process.

The minor impact of the lack of cpSRP54 might be explained with the hypothesis of a mRNA-based protein targeting. This mechanism is discussed to be potentially involved in targeting of proteins to the subcellular compartments (Nevo-Dinur et al., 2011; Weis et al., 2013). Likewise, the localization of the $p s b A$ mRNA at the thylakoid membrane might be important for protein delivery. Several $p s b A$ mRNA binding proteins have been described in A. thaliana and Chlamydomonas reinhardtii that are essential for D1 translation and possibly attach the $p s b A$ mRNA to the thylakoid membrane (Mulo et al., 2012). Another strong evidence for a mRNA-based targeting of D1 was found in $C$. reinhardtii. There, components of the translation machinery and the $p s b A$ mRNA accumulate in a specific region, called translation zone, independently of translation processes (Uniacke and Zerges, 2007, 2009).

In conclusion, our data indicate an almost cpSRP54independent targeting and insertion of D1 during the repair cycle of PS II, while cpFtsY plays an important role in this process. It is tempting to speculate that ribosomes might be associated with cpFtsY at the thylakoid membrane and that the psbA mRNA is delivered to the ribosomes in a translation-independent mechanism. However, it will be a major task for the future to elucidate the molecular details of cotranslational D1 insertion.

\section{Author Contributions}

DS and BW designed the research. BW and TP performed research. All authors contributed to data analyses and manuscript preparation.

\section{Acknowledgments}

This work was supported by a grant from the Deutsche Forschungsgemeinschaft (SCHU 1163/5-1). We thank Silke Funke for excellent experimental assistance, Neil Hoffman and Laurent Nussaume for the kind gift of chaos, ffc, and cpftsy seeds and Beatrix Dünschede for critically reading the manuscript.

Asakura, Y., Hirohashi, T., Kikuchi, S., Belcher, S., Osborne, E., Yano, S., et al. (2004). Maize mutants lacking chloroplast FtsY exhibit pleiotropic defects in the biogenesis of thylakoid membranes. Plant Cell 16, 201-214. doi: 10.1105/tpc.014787

Asakura, Y., Kikuchi, S., and Nakai, M. (2008). Non-identical contributions of two membrane-bound cpSRP components, cpFtsY and Alb3, to thylakoid biogenesis. Plant J. 56, 1007-1017. doi: 10.1111/j.1365-313X.2008.03659.x

Barkan, A. (1993). Nuclear mutants of maize with defects in chloroplast polysome assembly have altered chloroplast RNA metabolism. Plant Cell 5, 389-402. doi: $10.1105 /$ tpc.5.4.389

Bibi, E. (2012). Is there a twist in the Escherichia coli signal recognition particle pathway? Trends Biochem. Sci. 37, 1-6. doi: 10.1016/j.tibs.2011.09.004 
Bornemann, T., Jockel, J., Rodnina, M. V., and Wintermeyer, W. (2008). Signal sequence-independent membrane targeting of ribosomes containing short nascent peptides within the exit tunnel. Nat. Struct. Mol. Biol. 15, 494-499. doi: 10.1038/nsmb. 1402

Celedon, J. M., and Cline, K. (2013). Intra-plastid protein trafficking: how plant cells adapted prokaryotic mechanisms to the eukaryotic condition. Biochim. Biophys. Acta 1833, 341-351. doi: 10.1016/j.bbamcr.2012.06.028

Denks, K., Vogt, A., Sachelaru, I., Petriman, N. A., Kudva, R., and Koch, H. G. (2014). The Sec translocon mediated protein transport in prokaryotes and eukaryotes. Mol. Membr. Biol. 31, 58-84. doi: 10.3109/09687688.2014.907455

Franklin, A. E., and Hoffman, N. E. (1993). Characterization of a chloroplast homologue of the $54-\mathrm{kDa}$ subunit of the signal recognition particle. J. Biol. Chem. 268, 22175-22180.

Gu, S. Q., Peske, F., Wieden, H. J., Rodnina, M. V., and Wintermeyer, W. (2003). The signal recognition particle binds to protein L23 at the peptide exit of the Escherichia coli ribosome. RNA 9, 566-573. doi: 10.1261/rna.2196403

Herskovits, A. A., and Bibi, E. (2000). Association of Escherichia coli ribosomes with the inner membrane requires the signal recognition particle receptor but is independent of the signal recognition particle. Proc. Natl. Acad. Sci. U.S.A. 97, 4621-4626. doi: 10.1073/pnas.080077197

Kato, Y., and Sakamoto, W. (2009). Protein quality control in chloroplasts: a current model of D1 protein degradation in the photosystem II repair cycle. J. Biochem. 146, 463-469. doi: 10.1093/jb/mvp073

Klimyuk, V. I., Persello-Cartieaux, F., Havaux, M., Contard-David, P., Schuenemann, D., Meiherhoff, K., et al. (1999). A chromodomain protein encoded by the arabidopsis CAO gene is a plant- specific component of the chloroplast signal recognition particle pathway that is involved in LHCP targeting. Plant Cell 11, 87-99. doi: 10.1105/tpc.11.1.87

Lennartz, K., Plucken, H., Seidler, A., Westhoff, P., Bechtold, N., and Meierhoff, K. (2001). HCF164 encodes a thioredoxin-like protein involved in the biogenesis of the cytochrome b(6)f complex in Arabidopsis. Plant Cell 13, 2539-2551. doi: $10.2307 / 3871593$

Loschelder, H., Schweer, J., Link, B., and Link, G. (2006). Dual temporal role of plastid sigma factor 6 in Arabidopsis development. Plant Physiol. 142, 642-650. doi: $10.1104 /$ pp. 106.085878

Lu, B., and Hanson, M. R. (1996). Fully edited and partially edited nad9 transcripts differ in size and both are associated with polysomes in potato mitochondria. Nucleic Acids Res. 24, 1369-1374. doi: 10.1093/nar/24.7.1369

Luirink, J., von Heijne, G., Houben, E., and de Gier, J. W. (2005). Biogenesis of inner membrane proteins in Escherichia coli. Annu. Rev. Microbiol. 59, 329-355. doi: 10.1146/annurev.micro.59.030804.121246

Mulo, P., Sakurai, I., and Aro, E. M. (2012). Strategies for psbA gene expression in cyanobacteria, green algae and higher plants: from transcription to PSII repair. Biochim. Biophys. Acta 1817, 247-257. doi: 10.1016/j.bbabio.2011.04.011

Nevo-Dinur, K., Nussbaum-Shochat, A., Ben-Yehuda, S., and Amster-Choder, O. (2011). Translation-independent localization of mRNA in E. coli. Science 331, 1081-1084. doi: 10.1126/science.1195691

Nilsson, R., Brunner, J., Hoffman, N. E., and van Wijk, K. J. (1999). Interactions of ribosome nascent chain complexes of the chloroplast- encoded D1 thylakoid membrane protein with cpSRP54. EMBO J. 18, 733-742. doi: 10.1093/emboj/18.3.733

Nilsson, R., and van Wijk, K. J. (2002). Transient interaction of cpSRP54 with elongating nascent chains of the chloroplast-encoded D1 protein; 'cpSRP54 caught in the act.' FEBS Lett. 524, 127-133. doi: 10.1016/S0014-5793(02)03016-8

Nixon, P. J., Michoux, F., Yu, J., Boehm, M., and Komenda, J. (2010). Recent advances in understanding the assembly and repair of photosystem II. Ann. Bot. 106, 1-16. doi: 10.1093/aob/mcq059

Paila, Y. D., Richardson, L. G., and Schnell, D. J. (2014). New insights into the mechanism of chloroplast protein import and its integration with protein quality control, organelle biogenesis and development. J. Mol. Biol. 427, 1038-1060. doi: 10.1016/j.jmb.2014.08.016

Richter, C. V., Bals, T., and Schunemann, D. (2010). Component interactions, regulation and mechanisms of chloroplast signal recognition particle-dependent protein transport. Eur. J. Cell Biol. 89, 965-973. doi: 10.1016/j.ejcb.2010.06.020
Röhl, T., and van Wijk, K. J. (2001). In vitro reconstitution of insertion and processing of cytochrome $\mathrm{f}$ in a homologous chloroplast translation system. J. Biol. Chem. 276, 35465-35472. doi: 10.1074/jbc.M1030 05200

Rutschow, H., Ytterberg, A. J., Friso, G., Nilsson, R., and van Wijk, K. J. (2008). Quantitative proteomics of a chloroplast SRP54 sorting mutant and its genetic interactions with CLPC1 in Arabidopsis. Plant Physiol. 148, 156-175. doi: 10.1104/pp.108.124545

Schagger, H., and von Jagow, G. (1991). Blue native electrophoresis for isolation of membrane protein complexes in enzymatically active form. Anal. Biochem. 199, 223-231. doi: 10.1016/0003-2697(91)90094-A

Schleiff, E., and Becker, T. (2011). Common ground for protein translocation: access control for mitochondria and chloroplasts. Nat. Rev. Mol. Cell Biol. 12, 48-59. doi: 10.1038/nrm3027

Schult, K., Meierhoff, K., Paradies, S., Toller, T., Wolff, P., and Westhoff, P. (2007) The nuclear-encoded factor HCF173 is involved in the initiation of translation of the psbA mRNA in Arabidopsis thaliana. Plant Cell 19, 1329-1346. doi: $10.1105 /$ tpc. 106.042895

Schünemann, D. (2007). Mechanisms of protein import into thylakoids of chloroplasts. Biol. Chem. 388, 907-915. doi: 10.1515/BC.2007.111

Schünemann, D., Gupta, S., Persello-Cartieaux, F., Klimyuk, V. I., Jones, J. D., Nussaume, L., et al. (1998). A novel signal recognition particle targets lightharvesting proteins to the thylakoid membranes. Proc. Natl. Acad. Sci. U.S.A. 95, 10312-10316. doi: 10.1073/pnas.95.17.10312

Seluanov, A., and Bibi, E. (1997). FtsY, the prokaryotic signal recognition particle receptor homologue, is essential for biogenesis of membrane proteins. J. Biol. Chem. 272, 2053-2055. doi: 10.1074/jbc.272.4.2053

Träger, C., Rosenblad, M. A., Ziehe, D., Garcia-Petit, C., Schrader, L., Kock, K., et al. (2012). Evolution from the prokaryotic to the higher plant chloroplast signal recognition particle: the signal recognition particle RNA is conserved in plastids of a wide range of photosynthetic organisms. Plant Cell 24, 4819-4836. doi: $10.1105 /$ tpc.112.102996

Tzvetkova-Chevolleau, T., Hutin, C., Noel, L. D., Goforth, R., Carde, J. P., Caffarri, S., et al. (2007). Canonical signal recognition particle components can be bypassed for posttranslational protein targeting in chloroplasts. Plant Cell 19, 1635-1648. doi: 10.1105/tpc.106.048959

Uniacke, J., and Zerges, W. (2007). Photosystem II assembly and repair are differentially localized in Chlamydomonas. Plant Cell 19, 3640-3654. doi: 10.1105/tpc.107.054882

Uniacke, J., and Zerges, W. (2009). Chloroplast protein targeting involves localized translation in Chlamydomonas. Proc. Natl. Acad. Sci. U.S.A. 106, 1439-1444. doi: $10.1073 /$ pnas.0811268106

Weis, B. L., Schleiff, E., and Zerges, W. (2013). Protein targeting to subcellular organelles via MRNA localization. Biochim. Biophys. Acta 1833, 260-273. doi: 10.1016/j.bbamcr.2012.04.004

Yamamoto, T., Shimizu, Y., Ueda, T., and Shiro, Y. (2010). Mg2+ dependence of $70 \mathrm{~s}$ ribosomal protein flexibility revealed by hydrogen/deuterium exchange and mass spectrometry. J. Biol. Chem. 285, 5646-5652. doi: 10.1074/jbc.M109.081836

Zhang, L. X., Paakkarinen, V., Suorsa, M., and Aro, E. M. (2001). A SecY homologue is involved in chloroplast-encoded D1 protein biogenesis. J. Biol. Chem. 276, 37809-37814. doi: 10.1074/jbc.M105522200

Conflict of Interest Statement: The authors declare that the research was conducted in the absence of any commercial or financial relationships that could be construed as a potential conflict of interest.

Copyright (c) 2015 Walter, Pieta and Schünemann. This is an open-access article distributed under the terms of the Creative Commons Attribution License (CC $B Y)$. The use, distribution or reproduction in other forums is permitted, provided the original author(s) or licensor are credited and that the original publication in this journal is cited, in accordance with accepted academic practice. No use, distribution or reproduction is permitted which does not comply with these terms. 